\title{
A novel messenger RNA signature as a prognostic biomarker for predicting relapse in pancreatic ductal adenocarcinoma
}

\author{
Guodong Shi ${ }^{1,2, *}$, Jingjing Zhang ${ }^{1,2, *}$, Zipeng $\mathrm{Lu}^{1,2, *}$, Dongfang Liu ${ }^{1,2, *}$, Yang Wu ${ }^{1,2}$, \\ Pengfei Wu ${ }^{1,2}$, Jie Yinn ${ }^{1,2}$, Hao Yuan ${ }^{1,2}$, Qicong Zhu ${ }^{1,2}$, Lei Chen ${ }^{1,2}$, Yue Fu ${ }^{1,2}$, Yunpeng \\ Peng $^{1,2}$, Yan Wang ${ }^{3}$, Kuirong Jiang ${ }^{1,2}$ and Yi Miao ${ }^{1,2}$ \\ ${ }^{1}$ Pancreas Center, Department of General Surgery, The First Affiliated Hospital of Nanjing Medical University, Nanjing 210029, \\ China \\ ${ }^{2}$ Pancreas Institute of Nanjing Medical University, Nanjing 210029, China \\ ${ }^{3}$ Endoscopy Center, The First Affiliated Hospital of Nanjing Medical University, Nanjing 210029, China \\ *These authors contributed equally to this work \\ Correspondence to: Kuirong Jiang, email: jiangkuirongnjmu@sina.com \\ Yi Miao, email: miaoyi@njmu.edu.cn
}

Keywords: predictive signature; biomarker; bioinformatics; pancreatic ductal adenocarcinoma; relapse-free survival

Received: June 16, $2017 \quad$ Accepted: November 04, $2017 \quad$ Published: December 02, 2017

Copyright: Shi et al. This is an open-access article distributed under the terms of the Creative Commons Attribution License 3.0 (CC BY 3.0), which permits unrestricted use, distribution, and reproduction in any medium, provided the original author and source are credited.

\section{ABSTRACT}

Pancreatic ductal adenocarcinoma (PDAC) death rate and recurrence rate have remained obstinately high. Current methods can not satisfy the need of predicting cancer relapse accurately. Utilizing expression profiles of 10 GEO datasets $(N=774)$, we identified 154 differentially expressed genes (DEGs) between PDAC and normal pancreas tissue or paracancerous tissue. Next we built a 16-mRNA-based signature by means of the LASSO COX regression model. We also validated the prognostic value of the signature. Patients were classified into high-risk and low-risk group according to the signature risk score; 1 year RFS was 45\% (95\% CI: 31.6\%-63.9\%) for high-risk group in contrast to $92.5 \%$ (95\% CI: $86.3 \%-99.1 \%$ ) for low-risk group. Moreover, it could predict RFS well in cases with the receipt of different treatment modalities. The 16-mRNA-based signature was an independent and powerful prognostic biomarker for RFS for PDAC patients (HR = 7.74, 95\% CI: 3.25-18.45, $p<0.0001$ ).

\section{INTRODUCTION}

PDAC accounts for approximately $90 \%$ of pancreatic cancer (PC), which is the third leading cause of cancer mortality followed by lung cancer and colorectal cancer [1]. 5 year overall survival (OS) for PC has increased slightly from $5 \%$ to $8 \%$ and more than $52 \%$ cases are initially diagnosed at a distant stage for which 5 year OS is only $3 \%$ [1].The main reasons include the shortcomings of effective therapies and the lack of specific biomarkers or clinical symptoms in diagnosis.

Many therapeutic fields have emerged for decades, such as surgery, postoperative adjuvant chemoradiotherapy, targeted molecular therapy (TMT), neo-adjuvant chemotherapy, immunotherapy, and therapeutic exosomes or microvesicles.
Surgical resection is still the only potentially curative therapy. However, less than $20 \%$ have a surgical opportunity and over one-half of cases experience a postoperative relapse [2]. Cancer relapse directly results in shorter survival time after operation. Adjuvant chemotherapy with gemcitabine has been a standard care for resected PC which can delay recurrence [3].

Tumor tissue and serum samples contain massive potential diagnostic and prognostic biomarkers. Proteins and nucleic acid molecules including mRNAs, microRNAs, long non-coding RNAs and stable circular RNAs from tissue samples, and free molecules consisting of above mentioned molecules, tumor-derived exosomes or microvesicles, circulating tumor cells and circulating tumor DNA from serum samples have been explored as specific biomarkers for different tumors. 
Gene Expression Omnibus (GEO) and The Cancer Genome Atlas (TCGA) are two main public databases that provide massive array-based and sequence-based data for global researchers to download $[4,5]$. Meanwhile, novel bioinformatic methods make it quicker and more capable to deal with large amounts of data. To date, previous methods can't satisfied the need of predicting cancer relapse accurately. Although studies have identified some biomarkers for PDAC by integrating GEO with TCGA, most of them focused on overall survival instead of relapse-free survival. In our study, we carefully reviewed all datasets about PDAC and the eligible datasets were more comprehensive. We used $\mathrm{R}$ software to analyze gene expression levels and identify significant genes which expressed differentially between PDACs and normal pancreas tissue (TvsN) or paracancerous tissue (TvsP) [6]. Gene Ontology (GO) enrichment analysis and Kyoto Encyclopedia of Genes and Genomes (KEGG) pathway analysis were performed for finding out key genes and pathways. Furthermore, we utilized Least absolute shrinkage and selection operator (LASSO) regression model and built a 16-mRNA-based signature for predicting the present of relapse. The model was validated in 10-foldcross-validation [7]. The prognostic value of the signature was tested by the performance of multiple analyses.

\section{RESULTS}

\section{Identification of differentially expressed genes}

All eligible datasets were described in Table 1. Vocano plots of DEGs in these datasets were displayed in Figure 1. In addition, overlapping analysis of these DEGs was conducted. Four gene expression profiles were utilized to recognize DEGs of TvsN (Figure 2A). There were other 7 gene expression profiles used to identify DEGs of TvsP, including 3 gene expression profiles on agilent microarrays and 4 gene expression profiles on affymetrix microarrays (Figure 2B-2C). Due to the larger number of datasets, DEGs within 2 series or more were regarded as credible in each venn diagram. As shown in Figure 2D, there were 154 overlapping genes differently expressed among TvsN ( $\geq 2$ series), TvsP-affymetrix ( $\geq 2$ series) and TvsP-agilent ( $\geq 2$ series) finally. Of these, 103 genes were up-regulated mRNAs and 51 genes were down-regulated.

\section{Results of GO enrichment analysis and KEGG pathway analysis}

GO analysis and KEGG analysis were conducted for exploring the biological roles of these 154 DEGs. We listed top 10 terms in each of three categories (Figure 2E). Metabolic process was the main term of biological processes (BP), while terms associated with collagen and extracellular matrix (ECM) were enriched in cellular component (CC). Seven terms of molecular functions (MF) had relation with peptidase activity. As exhibited in Figure $2 \mathrm{~F}$, seven pathways were significantly enriched, of which protein digestion and absorption pathway was the most. The result of GO analysis integrated with KEGG analysis supported the point that ECM remodeling and pancreatic tumor-secreted microvesicles or exosomes promoted pre-metastatic niches formation and PDAC progression [8].

\section{Clinical characteristics of PDAC patients enrolled in the study}

One hundred and thirty-eight PDAC patients with RFS status were enrolled in our study. Clinical characteristics of these patients were summarized in Supplementary Table 1 . The median age of these patients was 65 years and median follow-up was 15.8 months. Thirty-nine of 138 patients experienced cancer relapse, of which 37 cases relapsed within 2 years. Following the AJCC 8th edition staging system, we had reassessed AJCC8 $\mathrm{T}$ stage, $\mathrm{N}$ stage and pathologic stage according to recorded maximum tumor dimension and the number of PLN (Supplementary Tables 2-4). Seventy-two T stage tumors, $46 \mathrm{~N}$ stage tumors and 62 pathologic stage tumors in the old system were reclassified into other stages in the 8 th version system.

\section{Construction and validation of the prognostic signature}

For these 154 candidate mRNAs, we used Nearest Neighbor Estimation (NNE) method to plot timedependent ROC curves. The predict time was equal to 24 months. We generated the optimal cutoff value and other indices of each mRNA, such as the area under the curve (AUC), Protective or Risky factor (PorR), sensitivity and specificity. According to the cutoff value, 138 patients were classified into high or low expression status of this mRNA. Next we conducted COX univariable analysis between each mRNA and RFS. These $p$ values were recorded. AUC $\geq 0.55$ was a restrictive condition for filtering some mRNAs that hardly had a prognostic value. Ultimately, $65 \mathrm{mRNAs}$ with $p<0.25$ and AUC $\geq 0.55$ were utilized to construct LASSO COX regression model (Supplementary Table 5). Of these, 53 genes were up-regulated mRNAs and 12 genes were down-regulated.

The function glmnet returned a sequence of models for us. We preferred the most widely used 10-fold cross-validation method to select the best one of them. As shown in Supplementary Figure 1, We plotted the partial likelihood deviance versus $\log (\lambda)$, where $\lambda$ was the tuning parameter. Herein, a value $\lambda=0.04513$ with $\log (\lambda)=-3.098$ was chosen by 10 -fold cross-validation via minimum criteria. Cross-validation was run up to 100 times. The cross-validated errors were averaged and 
Table 1: Datasets enrolled in the study

\begin{tabular}{lcccc}
\hline GEO ID or data source & T & P & N & Platform \\
\hline GSE62165 (Janky et al, 2016) & 118 & - & 13 & Affymetrix U219 \\
GSE91035 (Schmittgen et al, 2016) & 27 & 15 & 8 & Agilent LincRNA G3 \\
GSE32676 (Donahue et al, 2011) & 25 & - & 7 & Affymetrix U133 Plus 2 \\
GSE71989 (Schmittgen et al, 2015) & 13 & - & 8 & Affymetrix U133 Plus 2 \\
GSE62452 (Yang et al, 2016) & 69 & 61 & - & Affymetrix Gene 1.0 ST \\
GSE28735 (Zhang et al, 2012) & 45 & 45 & - & Affymetrix Gene 1.0 ST \\
GSE15471 (Badea et al, 2009) & 36 & 36 & - & Affymetrix U133 Plus 2 \\
GSE16515 (Pei et al, 2009) & 36 & 16 & - & Affymetrix U133 Plus 2 \\
GSE71729 (Moffitt et al, 2015) & 145 & 46 & - & Agilent G4112F \\
GSE58561 (Wennerström et al, 2014) & 3 & 2 & - & Agilent G3 \\
TCGA-PAAD (The Cancer Genome Atlas & 138 & - & - & Illumina HiSeq V2 \\
Research Network, 2014) & & & \\
\hline
\end{tabular}

N: normal pancreas tissue samples; P: paracancerous tissue samples; PAAD: patients with pancreatic adenocarcinoma; T: tumor samples.

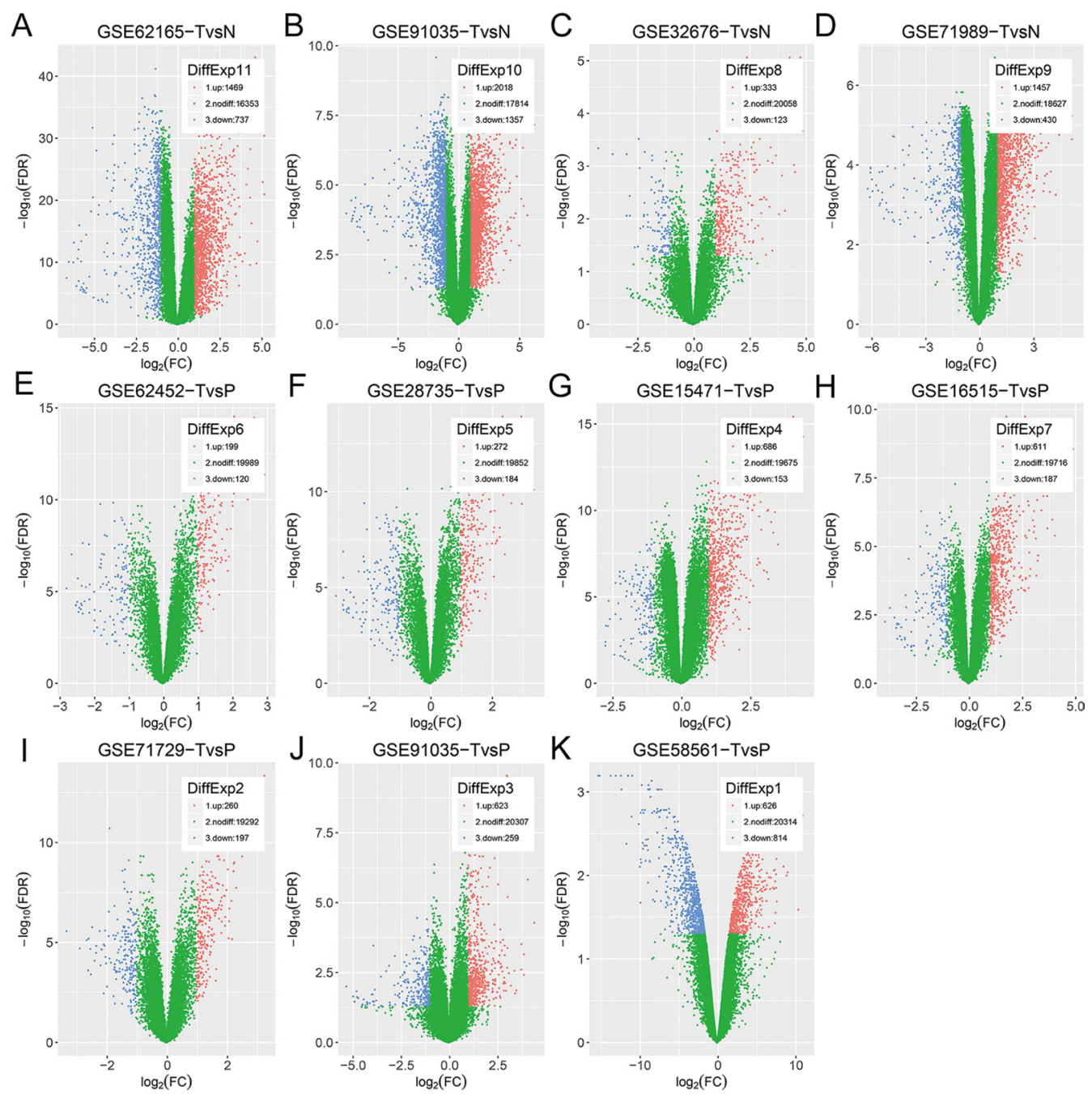

Figure 1: Vocano plots of DEGs in selected datasets. (A-K) GSE91035 consists of gene expression profiles of not only TvsN but also TvsP. X-axis: $\log [2](F C)$; Y-axis: $-\log [10]$ (FDR) for each gene. Genes with FDR $<0.05$ and absolute FC $>2$ are considered as DEGs in each series. Blue: down-regulated genes; Green: non-differential genes; Red: up-regulated genes. 
the lambda.min with minimum mean cross validation error was still equal to 0.04513 . So we obtained 16 variables with nonzero coefficients at the value $\lambda$ chosen by cross-validation. These mRNAs included ERP27, ASPM, ARNTL2, BIK, AMIGO2, SPOCK1, DKK1, COL17A1, MT1M, SERPINB5, CA4, FAM3B, MBOAT2, F11, ACSL5 and SLC4A4 (Figure 3A). On the basis of these mRNAs, we successfully built a 16-mRNA-based signature. The signature risk score of every patient was computed according to the summation of 16 mRNAs expression status multiplied coefficient: risk score of 16-mRNA-based signature $=\left(0.77120^{*}\right.$ status of ERP27 $)+$ $\left(0.62081^{*}\right.$ status of ASPM $)+\left(0.44536^{*}\right.$ status of ARNTL2 $)$ $+\left(0.40868^{*}\right.$ status of BIK $)+\left(0.30749^{*}\right.$ status of AMIGO2 $)+$ $\left(0.27687^{*}\right.$ status of SPOCK1 $)+\left(0.24414^{*}\right.$ status of DKK1 $)+$ $\left(0.24231^{*}\right.$ status of COL17A1 $)+\left(0.24071^{*}\right.$ status of MT1M $)$ $+\left(0.22296^{*}\right.$ status of SERPINB5 $)+\left(-0.01564^{*}\right.$ status of CA4 $)+\left(-0.02489^{*}\right.$ status of FAM3B $)+\left(-0.15373^{*}\right.$ status of MBOAT2 $)+\left(-0.16052^{*}\right.$ status of F11 $)+\left(-0.37751^{*}\right.$ status of ACSL5 $)+\left(-0.40839^{*}\right.$ status of SLC4A4). In this formula, Low expression status was equal to 0 and high expression status was equal to 1 .

The time-dependent ROC curve between the signature and RFS showed AUC at 2 years was 0.917 while AUC at 1 year was 0.817 (Figure 3B). It demonstrated that risk score value of 1.709 was the optimal cutoff point for predicting relapse, with sensitivity of 0.782 and specificity of 0.891 . According to the cutoff value, we classified patients into two different subgroups, of which low-risk group had lower risk score and high-risk group had higher risk score. Figure 3C showed Kaplan-Meier curve in which high-risk group had poor RFS. 1 year RFS was 45\% (95\% CI: $31.6 \%-63.9 \%$ ) for high-risk group and $92.5 \%$ (95\% CI: $86.3 \%-99.1 \%$ ) for low-risk group. Meanwhile, 2 year RFS was $0 \%$ for high-risk group in contrast to $87.4 \%(95 \% \mathrm{CI}$ : $78.6 \%-97.1 \%$ ) for low-risk group. The result of risk score analysis was displayed in Figure 3D. Besides, we noted that Youden's index (= sensitivity + specificity -1$)$ of 16-mRNA-
A

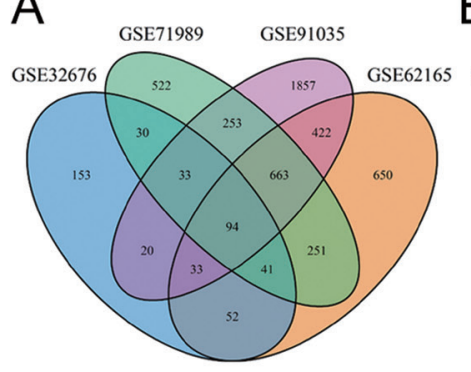

B

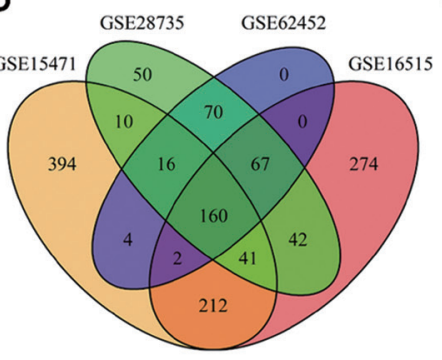

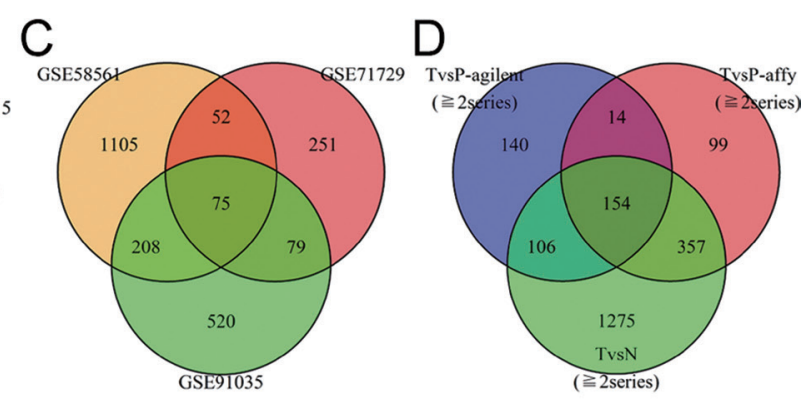

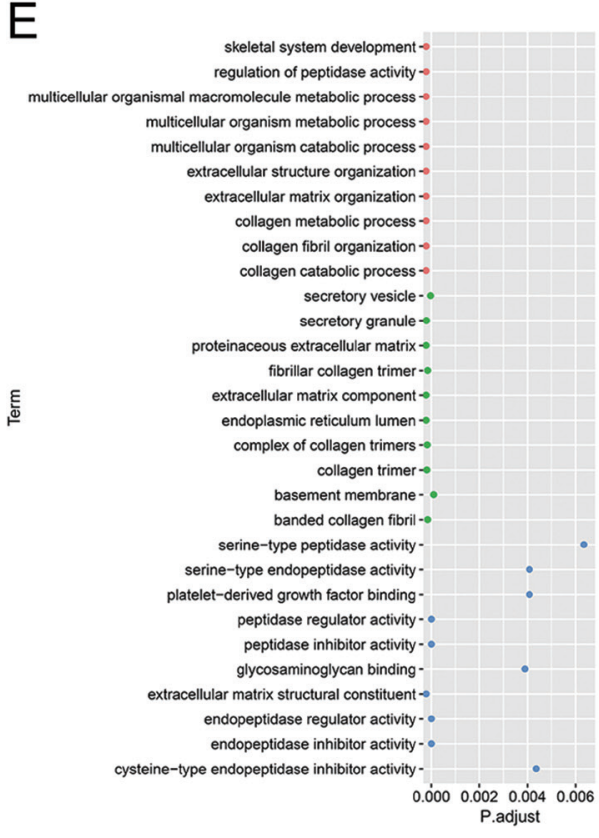

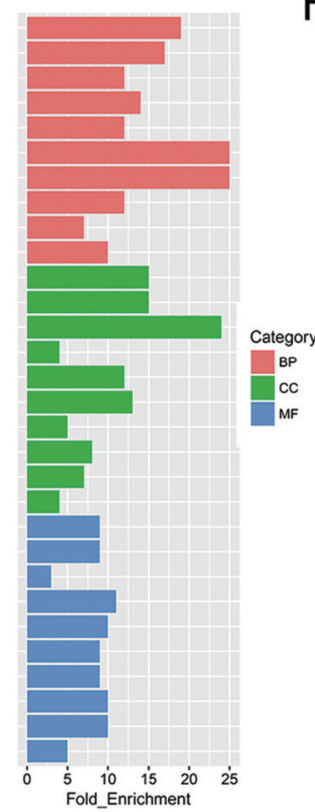

F

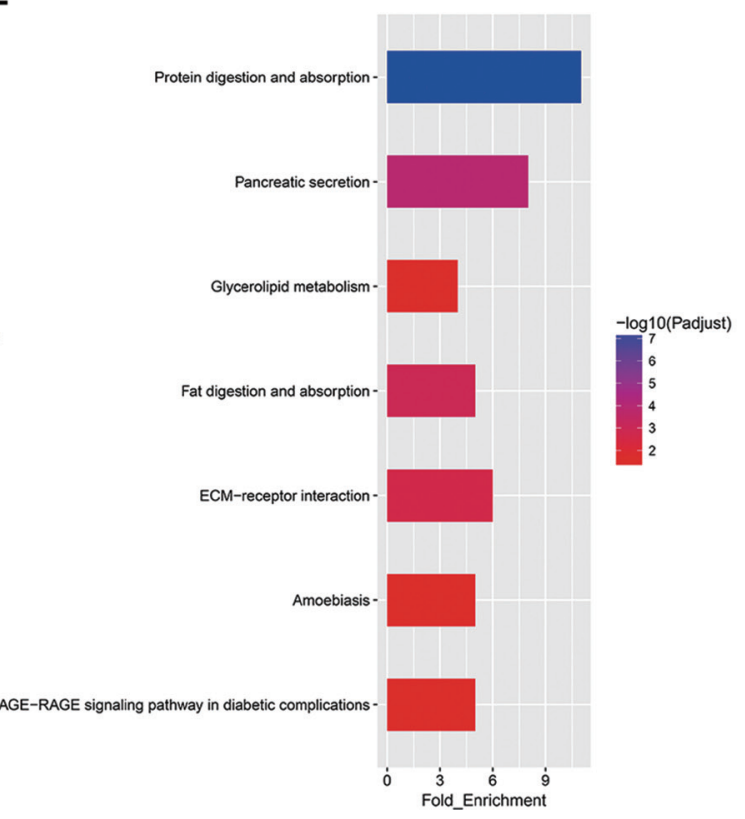

Figure 2: DEGs between PDAC and normal pancreas tissue or paracancerous tissue. (A-D) Overlap analysis in 11 different datasets. Every ellipse corresponds to a dataset. The numbers of DEGs in each overlapped area are marked in relevant position. DEGs within 2 dataset or more were regarded as credible in each venn diagram. (E) Top 10 terms in each of three categories of GO enrichment analysis. Red: biological processes (BP); Green: cellular component (CC); Blue: molecular functions (MF). (F) KEGG pathway analysis of DEGs. Seven signal pathways are significantly enriched. 
based signature was higher than any clinical factor or single mRNAs when predict time was equal to 24 months (Figure $3 \mathrm{E}-3 \mathrm{~F})$. Furthermore, we analyzed the association of the $16 \mathrm{mRNAs}$ and the signature risk score with all clinical characteristics (Supplementary Figure 2). We found that the distribution of tobacco smoking history, histologic grade, $\mathrm{T}$ stage and residual tumor status differed between high-risk and low-risk group.

\section{Results of COX univariable analysis, multivariable analysis and stratified analysis}

To verify that $16-\mathrm{mRNA}$-based signature had an excellent prognostic value, COX univariable analysis of 20 clinical factors and the signature with RFS were conducted. It demonstrated that 16-mRNA-based signature, histologic grade, $\mathrm{N}$ stage, $\mathrm{T}$ stage, primary therapy outcome success and AJCC8 $\mathrm{N}$ stage were significantly related to RFS $(p<0.05)$ (Table 2). We recognized that the hazard ratio for cancer relapse was 12.79 (95\% CI: 5.5-29.74) when highrisk group compared to low-risk group $(p<0.0001)$. All $p$ values were all calculated with Log-rank test here.

In the light of AJCC 8th version staging system's more repeatable estimation and finer stratification, we performed multivariable analysis adjusted by clinical factors with $p<0.15$ except AJCC 7th N stage and AJCC 7 th $\mathrm{T}$ stage (Table 2 ). The adjusted hazard ratio was 7.74 (95\% CI: 3.25-18.45, $p<0.0001$ ), which indicated that 16-mRNA-based signature was still an independent prognostic factor for PDAC patients.

After stratified by clinical factors, 16-mRNAbased signature remained powerful for predicting RFS (Supplementary Table 6). In patients with the receipt of different treatment modalities, the results returned that highrisk group would have worse RFS in those whether received radiation or TMT (Figure 4, Supplementary Table 6).
A
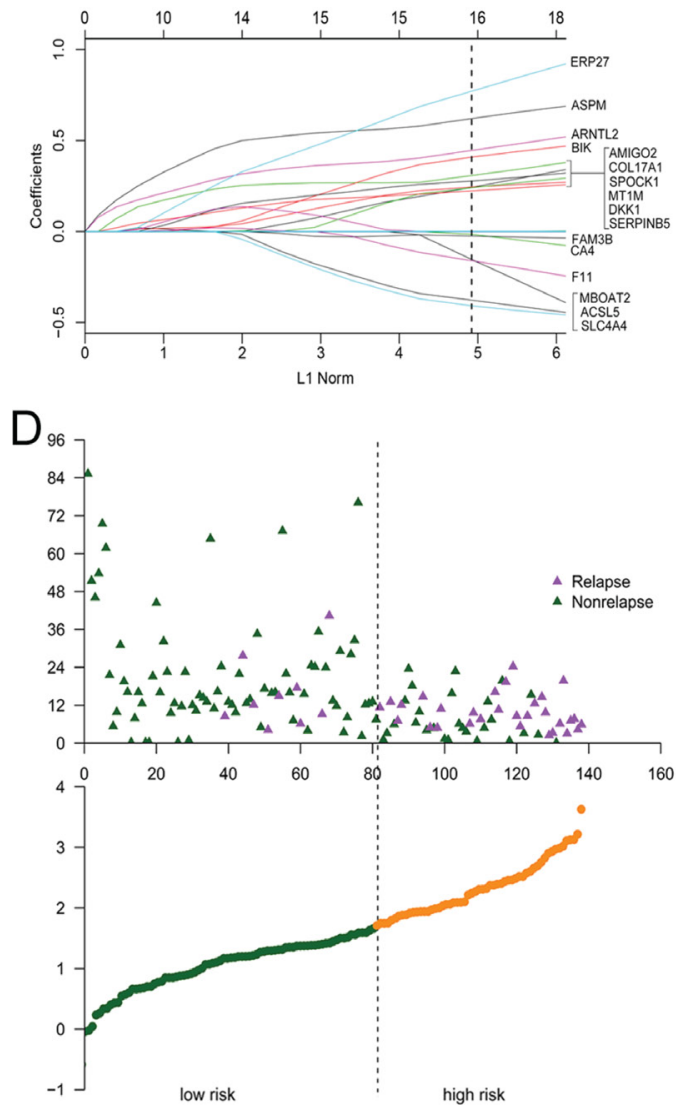

B
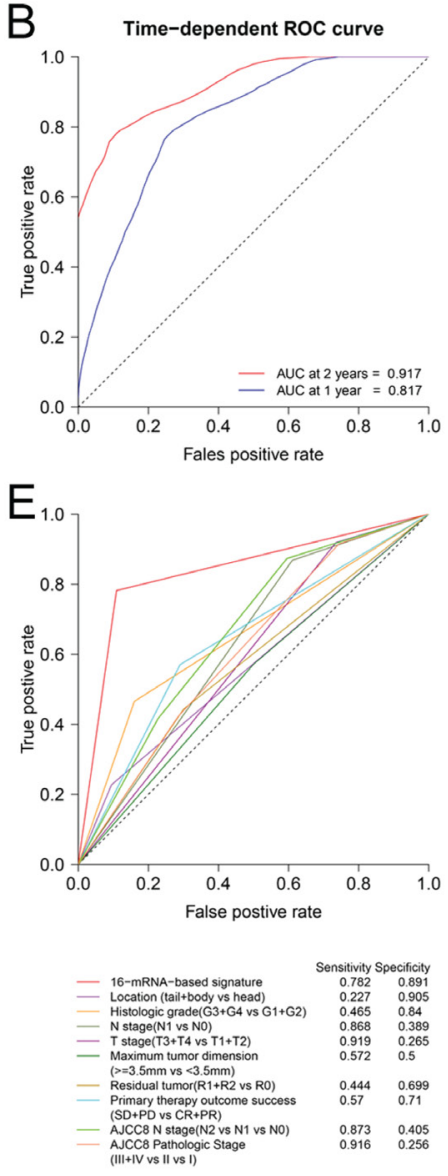

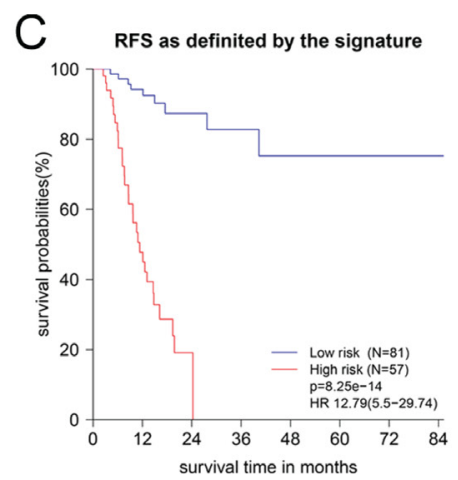

$\mathrm{F}$

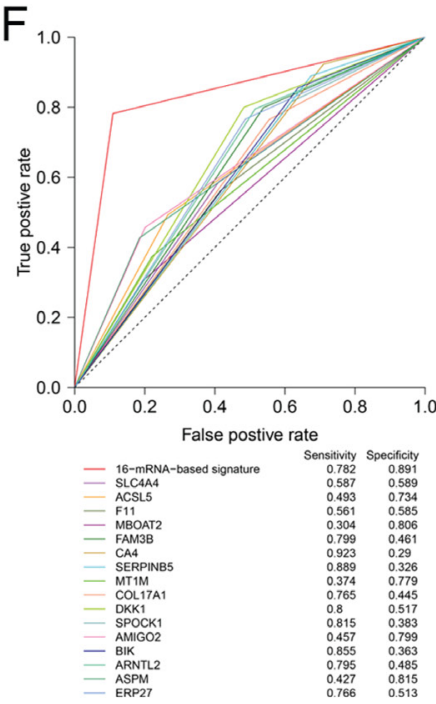

Figure 3: The 16-mRNA-based signature for predicting RFS. (A) Visualization of the coefficient profiles of fitting LASSO COX model. Each curve represents a variable. It shows the path of its coefficient against the L1-norm of the whole coefficient vector at as $\lambda$ varies. A vertical line is drawn at the value chosen by 10 -fold cross-validation. The above axis: the number of nonzero coefficients at as $\lambda$ varies. It represents the degrees of freedom for model. X-axis: L1 Norm, the summation of absolute nonzero coefficients at as $\lambda$ varies. Y-axis: the values of nonzero coefficients at as $\lambda$ varies. (B) Time-dependent ROC curve in 138 PDAC patients. (C) The KaplanMeier survival curve for patients in different risk groups. (D) Risk score analysis for patients. Top: the distribution of RFS status. Bottom: risk score of 16-mRNA-based signature. (E) Comparison of prognostic accuracy between the signature and clinical characteristics. (F) Comparison of prognostic accuracy between the signature and single mRNAs. 
Table 2: Result of COX univariable analysis and multivariable analysis of RFS

\begin{tabular}{|c|c|c|c|c|}
\hline Characteristics & $\begin{array}{l}\text { Univariable HR } \\
(95 \% \mathrm{CI})\end{array}$ & $P$ value & $\begin{array}{l}\text { Multivariable } \\
\text { HR (95\% CI) }\end{array}$ & $P$ value \\
\hline Gender (Female vs. male) & $1.2(0.64-2.26)$ & 0.5615 & & \\
\hline Age $(\geq 65$ years vs. $<65)$ & $1.01(0.54-1.92)$ & 0.9652 & & \\
\hline History of diabetes (YES vs. No) & $0.79(0.36-1.75)$ & 0.5578 & & \\
\hline History of chronic pancreatitis (YES vs. No) & $0.72(0.22-2.39)$ & 0.5943 & & \\
\hline Family history of cancer (YES vs. No) & $0.73(0.34-1.56)$ & 0.4186 & & \\
\hline Tobacco smoking history (YES vs. No) & $1.51(0.73-3.09)$ & 0.2625 & & \\
\hline Alcohol history documented (YES vs. No) & $1.39(0.7-2.79)$ & 0.3469 & & \\
\hline Location (tail + body vs. head) & $0.47(0.18-1.23)$ & 0.1161 & $0.35(0.1-1.23)$ & 0.1021 \\
\hline Histologic grade $(\mathrm{G} 3+\mathrm{G} 4$ vs. G1 + G2) & $2.54(1.34-4.84)$ & 0.0031 & $2.09(0.87-4.98)$ & 0.0973 \\
\hline Pathological stage (III + IV vs. II vs I) & $1.55(0.73-3.31)$ & 0.257 & & \\
\hline N stage (N1 vs. N0) & $3.14(1.3-7.56)$ & 0.0073 & & \\
\hline $\mathrm{T}$ stage $(\mathrm{T} 3+\mathrm{T} 4$ vs. $\mathrm{T} 1+\mathrm{T} 2)$ & $2.77(0.96-7.94)$ & 0.0497 & & \\
\hline Maximum tumor dimension $(\geq 3.5 \mathrm{~mm}$ vs. $<3.5 \mathrm{~mm})$ & $1.66(0.86-3.2)$ & 0.1304 & $1.57(0.71-3.51)$ & 0.2662 \\
\hline Residual tumor (R1 + R2 vs. R0) & $1.56(0.77-3.14)$ & 0.2095 & & \\
\hline Radiation therapy (YES vs. No) & $1.2(0.61-2.35)$ & 0.5895 & & \\
\hline Targeted molecular therapy (YES vs. No) & $1.14(0.54-2.41)$ & 0.7388 & & \\
\hline $\begin{array}{l}\text { Primary therapy outcome success (SD + PD vs. } \\
\mathrm{CR}+\mathrm{PR})\end{array}$ & $2.42(1.24-4.7)$ & 0.0074 & $1.31(0.61-2.83)$ & 0.4885 \\
\hline AJCC8th T stage (T3 + T4 vs. T1 + T2) & $1.37(0.73-2.58)$ & 0.3273 & & \\
\hline AJCC8th N stage (N2 vs. N1 vs. N0) & $1.59(1.06-2.39)$ & 0.0236 & $1.87(0.74-4.73)$ & 0.1828 \\
\hline AJCC8th Pathological Stage (III + IV vs. II vs I) & $1.49(0.95-2.32)$ & 0.0784 & $0.92(0.34-2.49)$ & 0.8699 \\
\hline 16-mRNA-based signature (High vs. Low) & $12.79(5.5-29.74)$ & $<0.0001$ & $7.74(3.25-18.45)$ & $<0.0001$ \\
\hline
\end{tabular}

AJCC: American Joint Committee on Cancer; CI: Confidence interval; CR: Complete remission /response; HR: Hazard ratio; PD: Progressive disease; PR: Partial remission/response; SD: Stable disease.

This was consistent with the result of Figure 3C. From Table 2, we noted that adjuvant radiation or TMT did not enhance survival in all patients $(\mathrm{HR}=1.2,95 \% \mathrm{CI}$ : 0.61-2.35; HR $=1.14,95 \%$ CI: 0.54-2.41, respectively). So we investigated the impact of radiation or TMT on RFS when these patients were stratified by 16-mRNA-based signature. However, we found no difference between radiation and non-radiation in whether high-risk group or low-risk group (Supplementary Figure 3A-3B). For low-risk group, it might be beneficial to treat with radiation, but might be bad to treat with TMT (Supplementary Figure 3A, 3C). They should receive postoperative radiation rather than TMT. High-risk group might potentially benefit from TMT, although the $p$ value did not reach statistical significance $(\mathrm{HR}=0.48,95 \% \mathrm{CI}: 0.20-1.14, p=0.09$, Supplementary Figure 3D).

\section{DISCUSSION}

Improving survival outcomes in PDAC patients is an arduous and urgent task, which requires the development of several aspects, such as early detection and precision diagnostic of tumorigenesis and metastasis, more advances in risk stratification with the help of biomarkers and imaging techniques, and multiple effective therapies available for individualized treatment.

In the present study, we established a novel mRNA signature as a prognostic biomarker for RFS. To obtain these 154 DEGs, we performed overlapping analysis with a strategical and stepwise method. The time-dependent ROC curve and COX univariable analysis initially evaluated the prognostic value of these DEGs. Sixty-five mRNAs with AUC $\geq 0.55$ and $p<0.25$ were utilized to construct LASSO COX regression model. Ten-fold cross-validation helped us to choose the best one with the minimum mean cross validation error from a sequence of models. The 16-mRNA-based signature risk score has been proven to be an independent and powerful prognostic factor for PDAC patients. Moreover, our risk score might be able to predict which patients benefit from TMT or radiation. Low-risk group might be suitable for treatment with radiation and high-risk group might potentially benefit from TMT.

Many clinical factors decreasing RFS after operation for PDAC were identified, such as elevated serum CA19-9 
$[9,10]$, perioperative blood transfusion $[11,12]$, higher histologic grade [9, 13], stage IV [11], lymph node metastasis [13-15] and lower lymphocyte to monocyte ratio [16]. However, unfavorable prognosis has not yet been improved. The present study showed that factors including histologic grade, AJCC7th N stage, AJCC7th $\mathrm{T}$ stage, primary therapy outcome success, AJCC8th $\mathrm{N}$ stage were associated with RFS. In term of PDAC staging, it is difficult to use imaging to distinguish extrapancreatic tissue from pancreas because of the proliferative reaction to tumor. To improve the repeatability and reproducibility of stage, AJCC staging system (8th edition) has canceled assessment of extrapancreatic invasion by using imaging as a criterion. Because 64 stage T3 tumors were downstaged to $\mathrm{T} 2(n=63)$ and $\mathrm{T} 1(n=1)$, the association between AJCC 8th T stage and RFS was not significant $(p>0.05)$. Besides, pathologic stage was not a prognostic factor for RFS $(p>0.05)$ in PDAC. This might be explained by that the distribution of subgroups was disproportionate and the sample size was not large enough. Nearly all patients had III stage tumors in stage III + IV group (94\%), and stage IIb patients accounted for $82 \%$ in stage II group. The malignant degree of tumor biology and survival between stage IIb and III patients were close. On the other hand, a non-significant interaction between pathologic stage and RFS was influenced by other factors possibly.

The prognostic potential of carbohydrate antigens has been well documented in recent years. Preoperative serum CA19-9 levels are the most widely used and the only biomarker approved by the U.S. Food and Drug Administration. Although it can serve as a diagnostic and prognostic biomarker, but its practical value is limited [9, 17-20]. Kohei et al. declared that preoperative serum CA19-9 level $(\geq 529 /<529 \mathrm{U} / \mathrm{mL})$ was an independent indicator for recurrence within 1 year after pancreatectomy. But it featured a poor prognostic value with sensitivity of $86.2 \%$ and specificity of $50 \%$ [9]. In contrast to CA19-9, the sensitivity and specificity of signature were $78.2 \%$ and $89.1 \%$ when predict time was two years. AUC at 2 years/1 year was $0.917 / 0.817$. To the best of our knowledge, there is little in the literature about the predictive value of other serum carbohydrate antigens for RFS. Since other serum carbohydrate antigens are inferior to CA19-9 in the early diagnosis of PDAC, Yu et al. confirmed that preoperative serum CA19-9 level $(\geq 37 /<37 \mathrm{U} / \mathrm{mL})$ had better predictive performance for OS and RFS than CA125 $(\geq 18.6 /<18.6 \mathrm{U} / \mathrm{mL})$ level in PDAC patients [19]. But CA125 is superior to CA19-9 in hyperbilirubinemia patients with resectable PDAC. From the time-dependent ROC curves, we observed that AUC at 2 years/1 year for CA19-9 was approximately 0.68/0.65, which was less than ones of the 16-mRNA-based signature. On the other hand, postoperative and post-adjuvant chemotherapy serum CA19-9 levels $(>37 / \leq 37 \mathrm{U} / \mathrm{mL})$ was all identified as an independent predictor for PDAC survival ( $\mathrm{HR}=2.697$ and 2.72, respectively), regardless of preoperative serum CA19-9 level [20, 21]. Moreover, elevated postoperative serum CEA $(>5.2 / \leq 5.2 \mathrm{ng} / \mathrm{mL})$ and CA125 (>35/ $\leq 35 \mathrm{U} / \mathrm{mL})$ level further decreased RFS [20]. It provides us a train of thought that we can monitor and combine postoperative serum CA19-9, CEA with CA125 levels to ameliorate risk stratification in pancreatic cancer after resection. Additionly, metabolic tumor burden measured by $18 \mathrm{~F}-\mathrm{FDG}$ PET/CT is an effective prognostic factor for RFS, and superior to preoperative serum CA19-9, but PET/CT examination is rather expensive [10]. Elevated ERCC1 $(\mathrm{HR}=2.1,95 \% \mathrm{CI}: 1.1-3.9)$ [22], DKK1 [23], CXCL12 [24], long non-coding RNA MALAT-1 (OR $=3.65,95 \%$ CI: $1.86-7.18)$ [25] in tissue sample, and detection of circulating tumor cells (pooled HR: 2.36, 95\% CI 1.41-3.96) [26] in peripheral blood were risky factors for patients survival. However, the 16-mRNA-based signature was significantly linked to RFS $(\mathrm{HR}=7.74,95 \%$ CI: 3.25-18.45).

Recent studies have proved that all the 16 mRNAs were differentially expressed between PDAC and normal pancreas tissue. Among these 16 mRNAs, 5 genes including ERP27, MT1M, CA4, F11 andFAM3B were down-regulated (Supplementary Table 5). ERP27 was
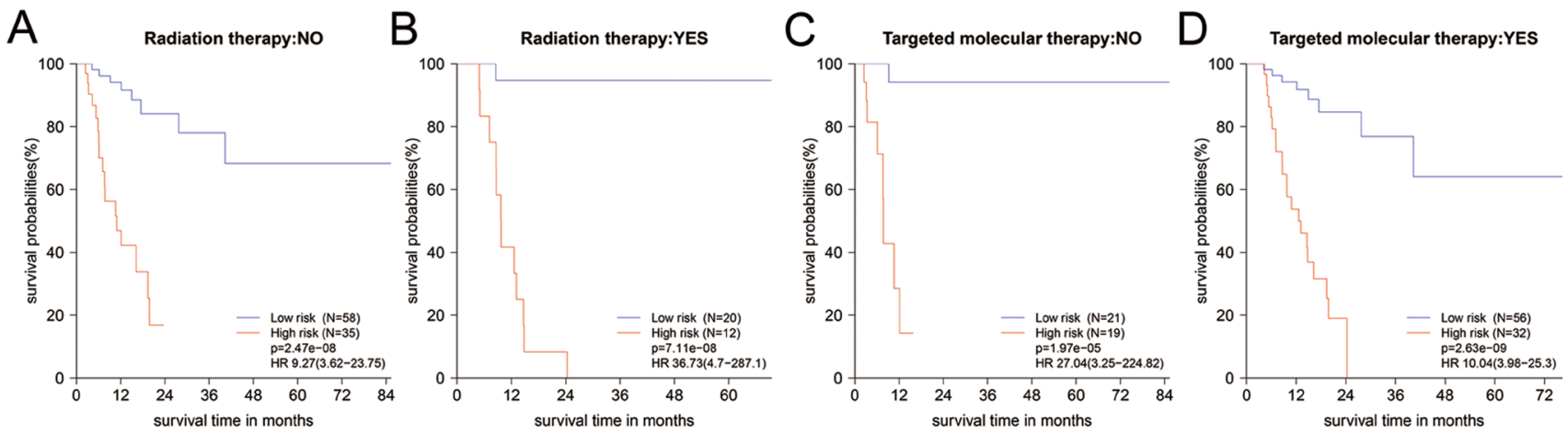

Figure 4: (A-D) The Kaplan-Meier survival curves for PDAC patients in different subgroups stratified by radiation and targeted molecular therapy. Information of partial patients on radiation or TMT was incomplete and deleted. TMT: targeted molecular therapy. 
shown to be down-regulated in acute pancreatitis in rats and selectively expressed in pancreatic exocrine glandular cells $[27,28]$. Tumor suppressor MT1M promoter methylation was utilized to detect hepatocellular carcinoma (HCC) non-invasively in blood [29]. Recent study firstly stated that CA4 was a tumor suppressor in colorectal cancer (CRC) by inhibiting the Wnt signaling pathway. CA4 promoter methylation was an independent biomarker for the recurrence of CRC [30]. Moreover, renal cell carcinoma patients after nephrectomy with lower CA4 had an adverse survival [31]. The expression level of F11 (coagulation factor XI) in normal pancreas is comparable with that in liver [32]. FAM3B also called pancreaticderived factor (PANDER) was secreted by pancreatic beta-cells and maintained glucose and lipid metabolism [33]. Another 11 genes were up-regulated. DKK1 was a WNT signaling pathway inhibitor whose promising diagnostic and prognostic value raised a continous concern in several tumors. It has been investigated that its high serological level was in connection with poor survival in CRC, laryngeal squamous cell carcinoma, HCC, lung cancer, esophageal carcinomas and PDAC [34-39]. It was also considered as a diagnostic biomarker in breast cancer and above mentioned cancers [34-40]. ASPM was connected with pancreatic epithelial tubulogenesis and over-expressed ASPM promoted aggressiveness of PDAC by regulating Wnt- $\beta$-catenin signaling pathway [41]. ARNTL2 was up-regulated and had the potential to be the marker for tumor aggressiveness in CRC [42]. $\mathrm{BIK}$, one of $\mathrm{Bcl}-2$ homology domain 3-only proteins was identified to induce apoptosis and predict breast cancer outcomes independently [43]. The pooled BH3only proteins served as a novel prognostic biomarker in glioblastoma multiforme [44]. Up-regulation of AMIGO2 in fibrosarcoma cells promoted liver metastasis through the development of liver endothelial cell adhesion [45]. SPOCK1 acted as a potential prognostic factor for pregression and took part in tumor proliferation and metastasis through the ERK and AKT signaling pathways [46]. The hyper-methylation of COL17A1 promoter increased ductal breast cancer metastasis. But COL17A1 was over-expressed and its promoter was hypo-methylated in cervical cancer and other epithelial cancers [47]. SERPINB5 (also called maspin) served as an independent risky prognosticator for OS in PDAC patients after operation [48]. Previous studies have announced that over-expression of MBOAT2 was negatively related to PDAC patient survival [49]. ACSL5 involved in enterocytic differentiation and maturation has already been a predictive prognostic factor for early tumor recurrence in CRC patients [50]. SLC4A4 mRNA involved in $\mathrm{Cl}(-)$ and $\mathrm{HCO}(3)(-)$ efflux was selectively expressed in pancreatic ductal cells higher than in islet cells $[28,51]$.

Using LASSO model, Zhang et al. built a sixmiRNA-based signature for identifying patients with stage II colon cancer who were suitable candidates for chemotherapy [52]. Ten-fold cross-validation was widely used to estimate how accurately a predictive model will perform in practice. The original samples were randomly classified into 10 equal sized subsamples, 9 subsamples were retained as training data while the remaining subsample was used as validation data. In this study, the 16-mRNA-based signature was at a minimum lambda with a minimum mean cross validation error. Transcript abundances of samples are got from RNA-seq rather than qRT-PCR. That is a limit of the study. But RNA-seq does not need to depend on reference gene for normalizing genes and is considered to be likely superior to qRTPCR in the future [53]. GAPDH and $\beta$-actin are usually selected as reference genes because of their constant and abundant expression level. However, the difference of GAPDH expression levels was observed between TCGA recurrent and non-recurrent group, whilst the expression level of $\beta$-actin did not vary significantly (Supplementary Figure 4). We can analogize that GAPDH expression level may have a difference between two groups in qRT-PCR even if all experimental processes are perfect. So the error will be also fatal when we perform qRT-PCR and select incorrect reference gene. qRT-PCR is not unchallengeable. The reasons of our study only chose the mRNAs were that many studies showed a wide difference in differentially expressed miRNAs (DEmiRs). The relation of long non-coding RNAs to PDAC survival has not been well documented. The extraction protocols and detection methods of mRNAs, miRNAs and long non-coding RNAs were also different.

In summary, the novel mRNA signature can be a prognostic biomarker and has an excellent accuracy of predicting cancer recurrence for postoperative patients.

\section{MATERIALS AND METHODS}

\section{PDAC datasets preparation}

Gene expression profiles of PDACs were downloaded from public GEO (https:/www.ncbi.nlm. nih.gov/geo/) and TCGA (http://cancergenome.nih. gov/). In the GEO Repository Browser, we retrieved 488 series with respective GSE identification numbers and a title containing the word "pancreatic". We exported all searching results into a Microsoft Excel and picked out 297 datasets about homo sapiens species. Their summaries were reviewed carefully and 17 GEO datasets about human gene expression profiles of TvsP or TvsN were eligible. Exclusion criteria were unanalyzable datasets; failure to meet quality control standards: actin3/actin5 $<3$ and gapdh3/gapdh5 $<1$ assessed by the function $q c$ in R package simpleaffy; the small number of of DEGs which was under 100; and incomplete annotated genes which accounted for less than $90 \%$ of genes within the total transcriptomes $(n<18000)$. Three datasets generated from non-mainstream platforms were unanalyzable and 
excluded. Four datasets have also been excluded due to other mentioned reasons (Supplementary Figure 5). In addition, TCGA database provided gene expression profile (level 3 data, $\log 2(\mathrm{RSEM}+1)$ transformed) from RNAseq and corresponding clinical information in 138 PDAC patients with RFS status.

\section{Gene expression profiles processing}

The raw cel files on affymetrix platform and the raw text file formats on agilent platform were gained. We used the function ReadAffy to read affymetrix microarray intensity data, and we conducted background correction, normalization and expression calculation with the function Robust Multi-Array Average (RMA) in the $\mathrm{R}$ package affy. Agilentmicroarray intensity data was read by using the function read.maimages in $\mathrm{R}$ package Linear Models for Microarray Data (LIMMA), and background correction, normalization and expression calculation were also completed by the package LIMMA. Boxplots were produced for observing whether scales of expression levels of microarrays were approximately equal. If multiple probes were annotated with the same gene, their mean value was computed to represent expression level of this common gene. The function Fit linear model (lmFit) in LIMMA was used to identify DEGs. Genes with adjusted $p$ value (Adj.P.Val, also called false discovery rate, FDR $)<0.05$ and absolute fold change $(\mathrm{FC})>2$ were considered as DEGs. R packages ggplot 2 were applied to draw Vocano plots for visualizing the results. Besides, overlapping genes were got from Venn diagram by means of the $\mathrm{R}$ package VennDiagram.

\section{GO enrichment analysis and KEGG pathway analysis}

We utilized 154 highly up-regulated or downregulated DEGs to perform GO enrichment analysis and KEGG pathway analysis in the $\mathrm{R}$ package clusterProfiler. Module categories with adjusted $p$ value $<0.05$ were identified to be enriched significantly.

\section{LASSO regression model building}

As we all know, Cox proportional hazards regression model is the most popular method of analyzing survival data. LASSO is a popular regression method and suitable for analyzing gene expression profile because microarray data has higher dimensionality, smaller sample size and strongly relevant variables [54]. Previous studies have applied it to Cox proportional hazards regression model broadly $[52,55]$. We constructed LASSO COX regression model by utilizing several DEGs which expressed abnormally in PDAC and were related to prognosis. The function glmnet in package glmnet returned a sequence of lambdas $(\lambda s)$ and models for us. The value of the tuning parameter $\lambda$ was negatively related to the complexity of the model and the value of deviance. As shown in Figure $3 \mathrm{~A}$, When the value of the invisible $\lambda$ increased from left to right, the number of nonzero coefficients increased accordingly, and L1 Norm, the summation of absolute nonzero coefficients would become bigger. By using the function cv.glmnet in package glmnet, 10-fold cross-validation was conducted for us to choose the best model. Even though it was so strict, the results of $c v$.glmnet were slightly variable. We run the function $c v . g l m n e t 100$ times follow the glmnet reference manual's advice and averaged cross-validation error curves. The lambda.min with minimum mean cross validation error was chosen by 10 -fold cross-validation via minimum criteria. Then we run the function glmnet or cv.glmnet once more with the lambda.min and extracted variables with nonzero coefficients and their corresponding coefficients. R codes and the input file for LASSO COX regression model were offered in Supplementary Tables 7-8.

\section{Statistical analysis and graphics}

All statistical analysis and graphics were performed in $\mathrm{R}$ software ( $\mathrm{R}$ version 3.3.2). Time-dependent ROC curves were designed to compute the optimal cutoff value, sensitivity and specificity by using the package survival ROC. Kaplan-Meier survival curves were drawn to analyze the relationship between variables and RFS in the survival package. We used Pearson chi-squared test, corrected chi-squared test or Fisher's exact test to examine the association of clinical characteristics with mRNAs. COX univariable analysis, multivariable analysis and stratified analysis were also performed in the package survival.

\section{Abbreviations}

Adj.P.Val: Adjusted $p$ value; AUC: Area under the curve; BP: Biological processes; CA199: Carbohydrate antigen 199; CC: Cellular component; CI: Confidence interval; CR: Complete remission/response; DEGs: Differentially expressed genes/mRNAs; DEmiRs: Differentially expressed miRNAs; ECM: Extracellular matrix; FC: Fold change; FDR: False discovery rate; GEO: Gene Expression Omnibus databases; GO: Gene Ontology; HCC: Hepatocellular carcinoma; HR: Hazard ratio; KEGG: Kyoto Encyclopedia of Genes and Genomes; LASSO: least absolute shrinkage and selection operator; LIMMA: Linear Models for Microarray Data; lmFit: Fit linear model; MF: Molecular functions; mRNAs: Messeger RNAs; NNE: Nearest neighbor estimation; OS: Overall survival; PC: Pancreatic cancer; PD: Progressive disease; PDAC: Pancreatic ductal adenocarcinoma; PLN: Positive lymph nodes; PorR: Protective or Risky factor; PR: Partial remission/response; RFS: Relapse-free survival; RMA: Robust Multi-Array Average; ROC: Receiver operating 
characteristic; SD: Stable disease; TCGA: The Cancer Genome Atlas database; TMT: Targeted molecular therapy; TvsP: Human PDACs versus paracancerous tissues; TvsN: Human PDACs versus normal pancreas tissues.

\section{ACKNOWLEDGMENTS AND FUNDING}

This work was supported in part by grants from the National Natural Science Foundation of China (81402489, 81572337 and 81672449), the Innovation Capability Development Project of Jiangsu Province (BM2015004), and the Priority Academic Program Development of Jiangsu Higher Education Institutions (PAPD, JX10231801). (Clinical Advanced Technology Program of Jiangsu Science and Technology Agency (BE2016788), Jiangsu Province's Key Provincial Talents Program (ZDRCB2016004), and Six Talent Peaks Project of Jiangsu Province (WSW-006)).

\section{CONFLICTS OF INTEREST}

The authors declare no conflicts of interest.

\section{REFERENCES}

1. Siegel RL, Miller KD, Jemal A. Cancer Statistics, 2017. CA Cancer J Clin. 2017; 67:7-30.

2. Sener SF, Fremgen A, Menck HR, Winchester DP. Pancreatic cancer: a report of treatment and survival trends for 100,313 patients diagnosed from 1985-1995, using the National Cancer Database. J Am Coll Surg. 1999; 189:1-7.

3. Oettle H, Post S, Neuhaus P, Gellert K, Langrehr J, Ridwelski K, Schramm H, Fahlke J, Zuelke C, Burkart C, Gutberlet K, Kettner E, Schmalenberg H, et al. Adjuvant chemotherapy with gemcitabine vs observation in patients undergoing curative-intent resection of pancreatic cancer: a randomized controlled trial. JAMA. 2007; 297:267-277.

4. Edgar R, Domrachev M, Lash AE. Gene Expression Omnibus: NCBI gene expression and hybridization array data repository. Nucleic Acids Res. 2002; 30:207-210.

5. Tomczak K, Czerwinska P, Wiznerowicz M. The Cancer Genome Atlas (TCGA): an immeasurable source of knowledge. Contemp Oncol (Pozn). 2015; 19:A68-A77.

6. Tippmann S. Programming tools: Adventures with R. NATURE. 2015; 517:109-110.

7. Ternes N, Rotolo F, Michiels S. Empirical extensions of the lasso penalty to reduce the false discovery rate in high-dimensional Cox regression models. Stat Med. 2016; 35:2561-2573.

8. Peinado H, Zhang H, Matei IR, Costa-Silva B, Hoshino A, Rodrigues G, Psaila B, Kaplan RN, Bromberg JF, Kang Y, Bissell MJ, Cox TR, Giaccia AJ, et al. Pre-metastatic niches: organ-specific homes for metastases. Nat Rev Cancer. 2017; 17:302-317.
9. Nishio K, Kimura K, Amano R, Yamazoe S, Ohrira G, Nakata B, Hirakawa K, Ohira M. Preoperative predictors for early recurrence of resectable pancreatic cancer. World J Surg Oncol. 2017; 15:16.

10. Xu HX, Chen T, Wang WQ, Wu CT, Liu C, Long J, Xu J, Zhang YJ, Chen RH, Liu L, Yu XJ. Metabolic tumour burden assessed by F-FDG PET/CT associated with serum CA19-9 predicts pancreatic cancer outcome after resection. Eur J Nucl Med Mol Imaging. 2014; 41:1093-1102.

11. Poorman CE, Postlewait LM, Ethun CG, Tran TB, Prescott JD, Pawlik TM, Wang TS, Glenn J, Hatzaras I, Shenoy R, Phay JE, Keplinger K, Fields RC, et al. Blood Transfusion and Survival for Resected Adrenocortical Carcinoma: A Study from the United States Adrenocortical Carcinoma Group. Am Surg. 2017; 83:761-768.

12. Kneuertz PJ, Patel SH, Chu CK, Maithel SK, Sarmiento JM, Delman KA, Staley CR, Kooby DA. Effects of perioperative red blood cell transfusion on disease recurrence and survival after pancreaticoduodenectomy for ductal adenocarcinoma. Ann Surg Oncol. 2011; 18:1327-1334.

13. Wentz SC, Zhao ZG, Shyr Y, Shi CJ, Merchant NB, Washington K, Xia F, Chakravarthy AB. Lymph node ratio and preoperative CA 19-9 levels predict overall survival and recurrence-free survival in patients with resected pancreatic adenocarcinoma. World J Gastrointest Oncol. 2012; 4:207-215.

14. Arima K, Hashimoto D, Okabe H, Inoue R, Kaida T, Higashi T, Taki K, Nitta H, Hayashi H, Chikamoto A, Beppu T, Baba H. Intraoperative blood loss is not a predictor of prognosis for pancreatic cancer. Surg Today. 2016; 46:792-797.

15. Aoyama T, Murakawa M, Katayama Y, Shiozawa M, Ueno M, Morimoto M, Yoshikawa T, Rino Y, Masuda M, Morinaga S. Lymphatic invasion is an independent prognostic factor in pancreatic cancer patients undergoing curative resection followed by adjuvant chemotherapy with gemcitabine or S-1. Hepatogastroenterology. 2015; 62:472-477.

16. Li W, Tao L, Zhang L, Xiu D. Prognostic role of lymphocyte to monocyte ratio for patients with pancreatic cancer: a systematic review and meta-analysis. Onco Targets Ther. 2017; 10:3391-3397.

17. Zhang Y, Jiang L, Song L. Meta-analysis of diagnostic value of serum Carbohydrate antigen 199 in pancreatic cancer. Minerva Med. 2016; 107:62-69.

18. Zhang Y, Yang J, Li H, Wu Y, Zhang H, Chen W. Tumor markers CA19-9, CA242 and CEA in the diagnosis of pancreatic cancer: a meta-analysis. Int J Clin Exp Med. 2015; 8:11683-11691.

19. Chen T, Zhang MG, Xu HX, Wang WQ, Liu L, Yu XJ. Preoperative serum CA125 levels predict the prognosis in hyperbilirubinemia patients with resectable pancreatic ductal adenocarcinoma. Medicine (Baltimore). 2015; 94:e751.

20. Xu HX, Liu L, Xiang JF, Wang WQ, Qi ZH, Wu CT, Liu C, Long J, Xu J, Ni QX, Yu XJ. Postoperative serum CEA and CA125 levels are supplementary to perioperative CA19-9 
levels in predicting operative outcomes of pancreatic ductal adenocarcinoma. Surgery. 2017; 161:373-384.

21. Imaoka H, Shimizu Y, Senda Y, Natsume S, Mizuno N, Hara K, Hijioka S, Hieda N, Tajika M, Tanaka T, Ishihara M, Niwa Y, Yamao K. Post-adjuvant chemotherapy CA19-9 levels predict prognosis in patients with pancreatic ductal adenocarcinoma: A retrospective cohort study. Pancreatology. 2016; 16:658-664.

22. Maithel SK, Coban I, Kneuertz PJ, Kooby DA, El-Rayes BF, Kauh JS, Sarmiento J, Staley CR, Volkan AN. Differential expression of ERCC1 in pancreas adenocarcinoma: high tumor expression is associated with earlier recurrence and shortened survival after resection. Ann Surg Oncol. 2011; 18:2699-2705.

23. Liu DJ, Xie YX, Liu XX, Huo YM, Yang MW, Fu XL, Liu W, Yang JY, Li J, Hua R, Liu PF, Sun YW, Zhang JF. The role of Dickkopf-1 as a potential prognostic marker in pancreatic ductal adenocarcinoma. Cell Cycle. 2017.

24. Samarendra H, Jones K, Petrinic T, Silva MA, Reddy S, Soonawalla Z, Gordon-Weeks A. A meta-analysis of CXCL12 expression for cancer prognosis. Br J Cancer. 2017; 117:124-135.

25. Wang Y, Xue D, Li Y, Pan X, Zhang X, Kuang B, Zhou M, Li X, Xiong W, Li G, Zeng Z, Yang T. The Long Noncoding RNA MALAT-1 is A Novel Biomarker in Various Cancers: A Meta-analysis Based on the GEO Database and Literature. J Cancer. 2016; 7:991-1001.

26. Ma XL, Li YY, Zhang J, Huang JW, Jia HY, Liu L, Li P. Prognostic role of circulating tumor cells in patients with pancreatic cancer: a meta-analysis. Asian Pac J Cancer Prev. 2014; 15:6015-6020.

27. Chen X, Sans MD, Strahler JR, Karnovsky A, Ernst SA, Michailidis G, Andrews PC, Williams JA. Quantitative organellar proteomics analysis of rough endoplasmic reticulum from normal and acute pancreatitis rat pancreas. J Proteome Res. 2010; 9:885-896.

28. Danielsson A, Ponten F, Fagerberg L, Hallstrom BM, Schwenk JM, Uhlen M, Korsgren O, Lindskog C. The human pancreas proteome defined by transcriptomics and antibody-based profiling. PLos One. 2014; 9:e115421.

29. Ji XF, Fan YC, Gao S, Yang Y, Zhang JJ, Wang K. MT1M and MT1G promoter methylation as biomarkers for hepatocellular carcinoma. World J Gastroenterol. 2014; 20:4723-4729.

30. Zhang J, Tsoi H, Li X, Wang H, Gao J, Wang K, Go MY, Ng SC, Chan FK, Sung JJ, Yu J. Carbonic anhydrase IV inhibits colon cancer development by inhibiting the Wnt signalling pathway through targeting the WTAP-WT1TBL1 axis. Gut. 2016; 65:1482-1493.

31. Takenawa J, Kaneko Y, Kishishita M, Higashitsuji H, Nishiyama H, Terachi T, Arai Y, Yoshida O, Fukumoto M, Fujita J. Transcript levels of aquaporin 1 and carbonic anhydrase IV as predictive indicators for prognosis of renal cell carcinoma patients after nephrectomy. Int J Cancer. 1998; 79:1-7.
32. Cheng Q, Kantz J, Poffenberger G, Powers AC, Gailani D. Factor XI protein in human pancreas and kidney. Thromb Haemost. 2008; 100:158-160.

33. Robert-Cooperman CE, Carnegie JR, Wilson CG, Yang J, Cook JR, Wu J, Young RA, Wolf BA, Burkhardt BR. Targeted disruption of pancreatic-derived factor (PANDER, FAM3B) impairs pancreatic beta-cell function. Diabetes. 2010; 59:2209-2218.

34. Kandimalla R, Linnekamp JF, van Hooff S, Castells A, Llor X, Andreu M, Jover R, Goel A, Medema JP. Methylation of WNT target genes AXIN2 and DKK1 as robust biomarkers for recurrence prediction in stage II colon cancer. Oncogenesis. 2017; 6:e308.

35. Shi Y, Gong HL, Zhou L, Tian J, Wang Y. Dickkopf-1 is a novel prognostic biomarker for laryngeal squamous cell carcinoma. Acta Otolaryngol. 2014; 134:753-759.

36. Shen Q, Fan J, Yang XR, Tan Y, Zhao W, Xu Y, Wang N, Niu Y, Wu Z, Zhou J, Qiu SJ, Shi YH, Yu B, et al. Serum DKK1 as a protein biomarker for the diagnosis of hepatocellular carcinoma: a large-scale, multicentre study. Lancet Oncol. 2012; 13:817-826.

37. Tung EK, Ng IO. Significance of serum DKK1 as a diagnostic biomarker in hepatocellular carcinoma. Future Oncol. 2012; 8:1525-1528.

38. Yamabuki T, Takano A, Hayama S, Ishikawa N, Kato T, Miyamoto $\mathrm{M}$, Ito $\mathrm{T}$, Ito $\mathrm{H}$, Miyagi $\mathrm{Y}$, Nakayama $\mathrm{H}$, Fujita M, Hosokawa M, Tsuchiya E, et al. Dikkopf-1 as a novel serologic and prognostic biomarker for lung and esophageal carcinomas. Cancer Res. 2007; 67:2517-2525.

39. Han SX, Zhou X, Sui X, He CC, Cai MJ, Ma JL, Zhang YY, Zhou CY, Ma CX, Varela-Ramirez A, Zhu Q. Serum dickkopf-1 is a novel serological biomarker for the diagnosis and prognosis of pancreatic cancer. Oncotarget. 2015; 6:19907-19917. http://doi.org/10.18632/oncotarget.4529.

40. Liu JT, Guo WB, Sun JY. Serum Dickkopf-1 acts as a new biomarker in human breast cancer. Minerva Med. 2017; 108:334-340.

41. Wang WY, Hsu CC, Wang TY, Li CR, Hou YC, Chu JM, Lee CT, Liu MS, Su JJ, Jian KY, Huang SS, Jiang SS, Shan YS, et al. A gene expression signature of epithelial tubulogenesis and a role for ASPM in pancreatic tumor progression. Gastroenterology. 2013; 145:1110-1120.

42. Mazzoccoli G, Pazienza V, Panza A, Valvano MR, Benegiamo G, Vinciguerra M, Andriulli A, Piepoli A. ARNTL2 and SERPINE1: potential biomarkers for tumor aggressiveness in colorectal cancer. J Cancer Res Clin Oncol. 2012; 138:501-511.

43. Lopez-Munoz E, Hernandez-Zarco A, Garcia-Hernandez N, Alvarado-Cabrero I, Zarco-Espinosa G, SalamancaGomez F, Arenas-Aranda D. BIK/NBK gene as potential marker of prognostic and therapeutic target in breast cancer patients. Clin Trans1 Oncol. 2012; 14:586-591.

44. Cartron PF, Loussouarn D, Campone M, Martin SA, Vallette FM. Prognostic impact of the expression/ 
phosphorylation of the BH3-only proteins of the BCL-2 family in glioblastoma multiforme. Cell Death Dis. 2012; 3:e421.

45. Kanda Y, Osaki M, Onuma K, Sonoda A, Kobayashi M, Hamada J, Nicolson GL, Ochiya T, Okada F. Amigo2upregulation in Tumour Cells Facilitates Their Attachment to Liver Endothelial Cells Resulting in Liver Metastases. Sci Rep. 2017; 7:43567.

46. Shu YJ, Weng H, Ye YY, Hu YP, Bao RF, Cao Y, Wang XA, Zhang F, Xiang SS, Li HF, Wu XS, Li ML, Jiang L, et al. SPOCK1 as a potential cancer prognostic marker promotes the proliferation and metastasis of gallbladder cancer cells by activating the PI3K/AKT pathway. Mol Cancer. 2015; $14: 12$.

47. Thangavelu PU, Krenacs T, Dray E, Duijf PH. In epithelial cancers, aberrant COL17A1 promoter methylation predicts its misexpression and increased invasion. Clin Epigenetics. 2016; 8:120.

48. Cao D, Zhang Q, Wu LS, Salaria SN, Winter JW, Hruban RH, Goggins MS, Abbruzzese JL, Maitra A, Ho L. Prognostic significance of maspin in pancreatic ductal adenocarcinoma: tissue microarray analysis of 223 surgically resected cases. Mod Pathol. 2007; 20:570-578.

49. Badea L, Herlea V, Dima SO, Dumitrascu T, Popescu I. Combined gene expression analysis of whole-tissue and microdissected pancreatic ductal adenocarcinoma identifies genes specifically overexpressed in tumor epithelia. Hepatogastroenterology. 2008; 55:2016-2027.

50. Hartmann F, Sparla D, Tute E, Tamm M, Schneider U, Jeon MK, Kasperk R, Gassler N, Kaemmerer E. Low acylCoA synthetase 5 expression in colorectal carcinomas is prognostic for early tumour recurrence. Pathol Res Pract. 2017; 213:261-266.

51. Hanzu FA, Gasa R, Bulur N, Lybaert P, Gomis R, Malaisse WJ, Beauwens R, Sener A. Expression of TMEM16A and SLC4A4 in human pancreatic islets. Cell Physiol Biochem. 2012; 29:61-64.

52. Zhang JX, Song W, Chen ZH, Wei JH, Liao YJ, Lei J, $\mathrm{Hu}$ M, Chen GZ, Liao B, Lu J, Zhao HW, Chen W, He YL, et al. Prognostic and predictive value of a microRNA signature in stage II colon cancer: a microRNA expression analysis. Lancet Oncol. 2013; 14:1295-1306.

53. Li B, Dewey CN. RSEM: accurate transcript quantification from RNA-Seq data with or without a reference genome. BMC Bioinformatics. 2011; 12:323.

54. Gui J, Li H. Penalized Cox regression analysis in the highdimensional and low-sample size settings, with applications to microarray gene expression data. Bioinformatics. 2005; 21:3001-3008.

55. Tibshirani R. The lasso method for variable selection in the Cox model. Stat Med. 1997; 16:385-395. 